\title{
Alzheimer's Disease and Related Dementias: A Qualitative Assessment of Perceived Training Needs of Primary Healthcare Providers in Rural Southwestern Uganda
}

Clara Decent Atuhaire ( $\sim$ clara@must.ac.ug )

Mbarara University of Science and Technology https://orcid.org/0000-0002-0998-7089

Celestino Obua

Mbarara University of Science and Technoogy

Godfrey Z. Rukundo

Mbarara University of Science and Technology

Jessica E. Haberer

Center for Global Health Research, Massachusetts General Hospital (MGH)

Edith K. Wakida

Office of Research Administration, Mbarara University of Science and Technology

\section{Research article}

Keywords: Alzheimer's disease, dementia, training needs, primary healthcare providers

Posted Date: September 20th, 2019

DOI: https://doi.org/10.21203/rs.2.14668/v1

License: (c) (i) This work is licensed under a Creative Commons Attribution 4.0 International License.

Read Full License 


\section{Abstract}

Background: Alzheimer's disease and related dementias (ADRD)are the third leading cause of years lived with disability, yet patients presenting with ADRD rarely receive proper care at primary healthcare centres (PHC). PHC providers are expected to be at the frontline of ADRD care and are often the first point of contact for patients with concerns about cognitive function; however, $\mathrm{PHC}$ providers may not have the knowledge and skills required to deliver person-centred care to patients. We therefore sought to identify the perceived training needs of PHC providers in recognizing, managing, and referring people with ADRD. Methodology: This was a cross-sectional qualitative study that was conducted in four districts in south western Uganda (Mbarara, Isingiro, Rukungiri, and Kabale). A semi-structured interview guide based on the WHO guide (toolkit) for community-based management and care of people with dementia was used to collect data from PHC providers (medical officers, clinical officers, nurses and midwives)districts. Results: The key training needs identified included awareness about ADRD among the PHC providers, knowledge on how to recognise, diagnose, manage and refer people with ADRD and in-service continuous medical education for ADRD, which would in turn lead to improved PHC providers' attitude towards elderly people with ADRD. Discussion:Results from the study show a lack of knowledge and inability to identify, diagnose and care for people with ADRD by PHC providers at HC III and HC IVs in rural South Western Uganda. However, The Kyoto Declaration of Alzheimer's Disease International (ADI) provides sufficient guidelines for streamlining dementia care in the primary healthcare system in low- and middle-income countries, which can be operationalized using the WHO toolkit. Conclusion: The lack of knowledge by PHCPs in rural south-western Uganda in identifying and managing persons with ADRD is an indication of the need to provide in-service training to improve the identification and management and sensitization of patients with signs of dementia. Key words: Alzheimer's disease, dementia, training needs, primary healthcare providers

\section{Background}

The global burden of disease studyestimates that Alzheimer's disease and its related dementias (ADRD)is the third leading cause of years lived with disability (1). Dementia affects not only the individual but also families and communities on personal, emotional, social, and financial levels, driving millions of households below the poverty line(2). About 47 million people globally are living with Alzheimer's disease and its related dementias (ADRD), of which $58 \%$ live in low- and middle-income countries (LMICs)(3). This number is projected to double every 20 years with an increasing burden in LMICs (4). In Africa, the rate of increase of in the size of the elder population is the fastest in the world. Uganda has a totalpopulation estimated at 42 million people with $4.6 \%$ aged 60 years and above(5). While the proportion of ADRD in the general population is unknown, a study of Ugandans aged 60 years and above admitted to nonpsychiatric wards found that $6.3 \%$ had dementia (6).In some communities, patients who present with dementia rarely receive proper care because the condition is regarded as part of the normal ageing process (7). 
Scanty information exists on what is involved in recognising and managing dementia especially in low resource settings $(8,9)$. Previous studies indicate that less attention has been paid to ADRD especially in the LMIC healthcare systems than other conditions because it is regarded as part of the normal ageing process $(3,7)$.

Additionally, there is scarcity of information about the primary healthcare $(\mathrm{PHC})$ providers knowledge and training in recognizing, treating or referring people with dementia (10) and yet they are typically at the frontline of dementia assessment and care (11).

Preparing PHC providers to meet the care needs of people living with ADRD requires deliberate and careful planning. During their training, these professionals are often not provided with the knowledge and skills required to deliver person-centred care for dementia (12).

We therefore set out to conduct a training needs assessment among PHC providers in southwestern Uganda to identify perceived gaps inrecognizing, providing care and management to people with ADRD. The needs assessment was based onthe World Health Organization (WHO) guidefor community-based management and care for people with dementia in LMICs(11).

\section{Methods}

\section{Study design}

This was a cross-sectional qualitative study that examined the training needs of PHC providers inrecognizing, providing care and management to people with ADRD. We used a semi-structured interview guide with questions adapted from the WHO guide for community-based management and care of people with dementia. The study was designed by CA in consultation with EKW, JH, GZR, and CO. The interview guide was pilot tested at a health facility that was not included in the study, and revisions made based on lessons learned.

The WHO guide aims to help community workers gain skills and build capacity in identifying people at risk for dementia and making referrals when necessary; providing support to people with dementia, their families and carers; and engaging local communities in dementia-related activities and contributing to the development of dementia-friendly communities in low- and middle-income countries(11). It includes (a) community resource checklist, (b) screening and detection tools, (c) tools for dementia management and care, (d) list of dementia prevention and promotion activities (e) monitoring and evaluation tools (f) pocket guide for carers.

\section{Study setting and context}

The study was conducted in Isingiro, Kabale, Mbarara and Rukungiri districts in rural southwestern Uganda. The data was collected from PHC providers at health centre (HC) III and IV facilities (sub-county and county level clinics, respectively). In Uganda, dementia is considered a mental health condition. Provision of mental health services begin at HC III facilities, with subsequent referrals to HC IV facilities, 
general hospitals, regional referral hospitals, and finally to the national referral hospital $(13,14)$. Each health facility level is expected to have general medical doctors (except in $\mathrm{HC} \mathrm{IIIs),} \mathrm{clinical} \mathrm{officers}$ (diploma level medical assistants), nurses and midwives, and psychiatric nurses. The HC Ills do not have general medical doctors but have all the other cadres of healthcare providers (15).

\section{Health Centre selection and participant recruitment}

Rural south western Uganda is comprised of two sub regions, Ankole region with 10 (ten) districts, and Kigezi region with 6 (six) districts. Purposively, two districts were selected from each region, based on the highest population of elderly people in any two districts from each of the two regions. Isingiro and Mbarara were picked from Ankole region, and Rukungiri and Kabale were picked from Kigezi region.

At district level, selection of the Heath facilities was done using proportionate sampling. Based on the(16, 17)reports, it was established that there are 75 Health facilities (19 HC IVs and $56 \mathrm{HC}$ IIIs) in the 4 selected districts. In consultation with the $\mathrm{DHO}$ at each district, $\mathrm{CA}$ generated a list of all the $\mathrm{HC}$ Ills and HC IVs from where we screened for desired characteristics, and criteria for inclusion in the study was; if they were a government facility; had PHC providers who directly assessed patients, and were not neighbouring or not located within 20 kilometres of other health facilities or a hospital (otherwise the elderly would seek medical care from the hospital, which is outside the PHC setting). The rationale for selection of HCs far away from other healthcare providers meant that there was a higher chance that the elderly people could only access healthcare from these PHC facilities nearest them, increasing the chances that all PHC providers will have interacted with many of them including the elderly at risk of ADRD. Based on this criterion, $6 \mathrm{HC}$ Ills and $6 \mathrm{HC}$ IVs were selected from Ankole sub-region, and $6 \mathrm{HC}$ IVs and $6 \mathrm{HC}$ Ills were selected from Kigezi sub-region (Figure 1). The study participants included medical doctors, clinical officers, nurses and midwives from the twenty-four HCs (12 HCs III and $12 \mathrm{HC} \mathrm{IVs).At}$ each selected health facility, participants were recruited since they directly assess patients, and for each cadre level, respondents were interviewed until saturation occurred.

\section{Data collection procedure}

In-depth interviews were conducted by the lead author (CA) and two trained research assistants in January 2019. Each interview lasted approximately 60 minutes and was audio recorded. All interviews were conducted in English (the official national languagetypically used in medical settings) and supported by field notes.

\section{Data collection and tools}

A semi-structured interview guide wasdeveloped by CA in consultation with EKW, CO, and $\mathrm{JH}$. The questions were adapted from the WHO guide for Community-Based Management and Care of people with dementia(11). The interview guide was pilot tested at a health facility that was not included in the study.

\section{Data management and analysis}


Data was transcribed verbatim by the research assistants, verified by an independent transcriber, and then crosschecked by CA against the audio recordings for correctness of information after each of interviews. Using the first set of transcripts, clarification was sought from EKW, CO, and JHto ensure that the questions asked, and responses were done in the correct way and would answer the research questions. CA, and EKW independently read through transcripts and developed codes in accordance with emergent themes; the initial coding was done by CA, and codified by EKW in consultation with CA.Data werethematically analysed (18) with the help of a qualitative software Atlas.ti version 7 (19).

\section{Ethical considerations}

The study was approved by the Mbarara University Research Ethics committee (MUREC) and the Uganda National Council of Science and Technology (UNCST). Permission to conduct interviews in the HCs was obtained from the respective District Health Officers. All participants provided written informed consent before each in-depth interview. No participant withdrew from the study. All the audio recorded material and transcripts were safely storedby to the lead author (CA).

\section{Results}

Fourthemesemerged from our data: (1) Inadequate awareness about ADRD, (2) Limited ability of PHC providers to Identify and diagnoseADRD in the elderly, (3) Lack of Training (continuous medical education) in Alzheimer's and related dementias, and (4) Poor attitudes of PHC providers towards people with ADRD.

A total of 51 in-depth interviews were conducted which was sufficient to achieve thematic saturation. We had more females $(n=30)$ than males $(n=21)$. We observed understaffing at $\mathrm{HC}$ Ills and as such more participants were interviewed from HC IVs (34/51) than from HC IIIs (17/51). The age ranged from 20 and 55 years (Table 1). Of the participantsinterviewedthere were 30nurses, eight midwives, three psychiatric nurses, 11 clinical officers, and two medical officers in the health facilities. Views of all the participants were included in the analysis and contribute to the conclusions in our study.

\section{Awareness about Alzheimer's disease and related dementias}

When asked about awareness of ADRD, participants generally described ADRD as a degenerative condition of the elderly associated with forgetfulness.

"... is a degenerative disorder that occurs in mostly the elderly with features of dementia they have what we call tremors, most of them tend to forget the near things than the far; they usually tend to have a crystal idea of the past but the near they usually forget" (Medical Officer, Isingiro district).

Theparticipantsreported that they were aware of other forms of dementia, such as that induced by HIV, alcoholism, drug and substance abuse, nutrition, as well as the environment induced dementia that results from stress. 
Uhhhmmmm... it can be trauma, it can also be usual conditions like meningitis, HIV, some people can get that syndrome and also maybe age (Clinical Officer, Kabale district)

In cases of head injuries, dementia can result, in cases of a person with epilepsy and it is poorly treated can also develop dementia and also cases of infections, meningitis and deficiencies in Vitamin - VitB1, VitB12 can also lead to dementia. It can also be genetic in other instances (Psychiatric Nursing Officer, Isingiro District).

When probed for the symptoms of ADRD, PHC providers reported looking fortremors, memory loss, complete lack of self-care, and hallucinations.

You find those people presenting with tremors; they forget quickly, they hallucinate, and that is why they are sometimes referred to the psychiatric department because they have psychiatric symptoms (Nursing Officer, Isingiro District)

There is loss of memory, they are disoriented, the concentration is poor, you find the sleep is also very poor (Principle Nursing Officer, Kabale district)

\section{Identifyingand diagnosing dementia in the elderly}

Participants indicated that the earliest opportunity for the PHC providers to identify elderly people with dementia is when the mental functions start to fail -that the elderly person starts to forgetand there is disorientation.

Definitely when they come and you try to ask them about their ages and they cannot remember when they were born and some events that could have happened when we are taking history, then you realize that this person could be having a problem (Clinical Officer, Mbarara district)

When you communicating to that client and you want them maybe to paraphrase what you have said that person forgets the story you have been talking about and...even when you put something some where they can look around the whole house looking for where they have placed their items and they fail to remember where they put them. So, you know that they have such a problem. (Nursing Officer, Mbarara district)

Participants also indicated that they used the physical hygiene of the person to determine that they had ADRD. They noted that if the elderly patients' ways of dress and conduct had drastically changed and they looked like they were not aware about what was going on, they would asses for ADRD. One participant said,

The cognitive aspect of it - if they are not taking care of themselves - their personal hygiene declines, where they are unkempt, they cannot look afterthemselves. That is when we can assess. (Clinical Officer, Rukungiri district) 
Our study also found that somepatients who had a long-standing relationship with PHC providers often disclosed difficulty to recall recent things as expressed by a nursing officer in one of the districts:

"Musaawo[Nurse],...the other day I forgot to wear clothes, and it is my kids who alerted me and said, Mummy, please!! Put on clothes.....so they tell us" (Nursing Officer, Mbarara district)

When asked how they concluded that someone has ADRD, most of the participants indicated that they had not been equipped with the necessary skills to recognize and diagnose Alzheimer's disease and related dementias. Therefore, they were not diagnosing it for fear of misdiagnosing.

I have not made a diagnosis of Alzheimer's. What I can only do is treat symptoms or signs or making other diagnosis, sometimes calling them dementia or psychosis. And most of the patients we treat here, we treat for psychosis. So, I do not know whether sometimes we make the wrong diagnosis, but most possibly it is oftentimes the wrong diagnosis. (Clinical Officer, Rukungiri district)

During the study, we found that there were no guidelines at the health facilities to support the PHC providers when screening for ADRD. Most of the participants expressed the need for some form of criteria to guide them when attending to the elderly patients as expressed by a clinical officer in Mbarara district.

We do not have criteria of scoring people to know those signs of forgetfulness and those other related signs (Clinical Officer, Mbarara district)

\section{Trainingin Alzheimer's and related dementias}

When we asked the participants if they thought they needed training in ADRD, most answered to the affirmative. They indicated that PHC providers needed to know more about the condition and how to manage it, including where to refer patients for specialized care.

Generally, people take dementia to be a simple disease. They don't take it as a serious problem. But if we could get to know more about it, perhaps we can take it seriously. And if we can get to know where to refer, it would help, because for us we think that a psychiatric disease is referred when someone is boxing people or when they have to be tied with ropes [restrained]. But for someone who is just forgetting things and you refer them [says this laughing], they do not take you seriously when they see such a referral. (Clinical Officer, Isingiro district)

The participants reported not receiving any in-service training related to ADRD. They suggested that to improve management of many diseases, the $\mathrm{PCH}$ providers should be provided with continuous medical education.

I have not had training in dementia management, butl met them in the normal training...for us to improve in management of many diseases, we should be provided with CMEs (Nursing Officer, Isingiro district)

\section{PHC provider's attitudes}


When asked what they would do when they got a patient with signs ADRD, most of the participants did not have an obvious response because they believed that some of the signs like forgetfulness were part of the normal aging process. They indicated that they would instead concentrate on more obvious symptoms as illustrated below:

Do I even know? When they come, we treat those other problems- neuropathy, high blood pressure-so we have not been concentrating on dementia. Maybe if the person is admitted and you see that this person is old, and the treatment schedule you emphasize it, maybe to the daughter or son who is taking care of the patient, because you think that this person is old and everything I tell him he may not be able to remember but having a mind that let me look out for dementia, ....(laughs).... I have not been doing that. (Clinical Officer, Isingiro district)

Concerning referring and receiving patients with ADRD, participants acknowledged the presence of community linkage facilitators, but decried their limited capacity. They added that although they received referrals through the community linkages, they hardly received those related to dementia.

...we also have an issue of lack of community linkage - we have community linkage facilitators but they basically major in a few things - ...HIV, PMTCT. Those referrals we get but it is difficult to get a psychiatric related referral (dementia), because people take it normal that anyone old should forget. So, they tend to look at it as something normal (Nursing Officer, Mbarara district).

\section{Discussion}

\section{Identifying and diagnosing ADRD in the elderly}

Results from the study show a lack of knowledge and inability to diagnose or manage dementia at healthcentres in the community. The PHC providers described what they thought was ADRD, but the level of knowledge varied among the participants. While the PHC providers could assess and determine that the elderly person might be having dementia, they refrained from giving a diagnosis for fear of making a wrong diagnosis(18). This, in turn presented a challenge for the PHC providers in the management and care for patients with signs of ADRD because of the limited competence in the area.

During a conference of Alzheimer's Disease International (ADI) in 2004 in Kyoto(the ADIKyoto Declaration, at which ten key areas for action were recommended to address Alzheimer's Disease in LMIC(26). Importantly was the need to recognize dementia care as a component of $\mathrm{PHC}$, to include the recognition and treatment of dementia in the training curricula of all health personnel,and to provide refresher training to PHC providers among other areas. However, a gap between this goal and routine clinical care remains as PHC providers in LMICs are not well equipped to give a reliable diagnosis of ADRD (18). This assessment agrees with the work of Montgomery et al (2014) in Nepal, as well as other research elsewhere (20) that the medical practitioners had insufficient knowledge to effectively diagnose, manage and refer people with dementia. 


\section{Trainingin Alzheimer's and related dementias}

Our study confirms a knowledge gap in the management and care of people with ADRD by the PHC providers at HCs III and IV in rural south western Uganda. All healthcare cadres irrespective seniority expressed the need for in-service training to gain the competencies in the management and care of people with dementia in the community. This result aligns with the evidence that health practitioners are not adequately prepared to handle patient's dementia, and as a result, they focus on the opportunistic ailments when faced with the elderly patients and miss the dementia diagnosis $(21,22)$.

Equipping the PHC providers with the necessary skills in the management and care of people with dementia will not only contribute to the ADI Kyoto declaration recommendation to give care in the community but will contribute to the quality of life of the elderly. This is premised on the principle that people with dementia are best assessed and treated in their own homes(23). It suffices to add that in addition to the training that the PHC practitioners need, there is also need to conduct continuous medical education (CMEs) sessions in order to continuously bring the PHC providers up-to-date with findings that come in from the global research fora and policy recommendations.

Pucci et al, 2003 (9)reported that during a training to determine the capacity of Italian general doctors to recognise and diagnose ADRD the pre-training results indicated that the general practitioners lacked sufficient knowledge in order to diagnose and manage dementia, a report not very different from what we found among the study participants. This shows that indeed there is need for training PHCPs to be able

to handle the diagnosis and management of ADRD at the lower health facility levels. Keeping in mind the fact that the line ministries face a lot of competing demands from what is nationally regarded as a priority, there is need to design simple interventions that can make a difference in the quality of life, if better education and healthcare systems were in place. Overall, trainings can be organised as a one-off for all PHC providers and thereafter CMEs which are cost effective(24), thus bring the PHC providers upto-date with required information.

\section{Limitations}

The research was conducted in PHC setting in four districts in south-western Uganda and may not be generalizable to other settings. There may be need for similar studies in the other regions to confirm our findings.

\section{Conclusions}

PHC providers in rural south-western Uganda exhibitedknowledge gaps in identifying and managing persons with ADRD, and in additionthere was expressed the need forin-service training as a meansto improve the diagnosis, management and referral of persons who present with symptoms of dementia at the primary healthcare facilities. Thus, calling for interventional training of the PHC providers inmore districtsin the country with implications for other LMIC countries. 


\section{Abbreviations}

ADRD Alzheimer's Disease and Related Dementias

CHWs Community Health Workers

HC Health centre

LMICs Low- and Middle- Income Countries

PHC Primary Healthcare

WHO World Health Organisation

\section{Declarations}

\section{Acknowledgements}

The research team would like to acknowledge the invaluable contribution of the Research Assistants, Andrew Katongole, Joan Namuli and Angella Babirye, who supported the author in data collection. Special regards also extend to the District Health Officers in the participating districts who granted the author permission to conduct the study in their districts. Above all, the research group gratefully acknowledges the time, expertise and experience from the participants.

\section{Funding}

Research reported in this publication was supported by the Fogarty International Center and the National Institute on Aging of the National Institutes of Health under Award Number D43TW010128. The content is solely the responsibility of the authors and does not necessarily represent the official views of the National Institutes of Health

\section{Availability of data and materials}

Data on which this manuscript is based will not be publicly available sincethis work is still ongoing but will be available in future when the intervention component is completed.

\section{Authors' contributions}

All authors contributed to the design of the study, data analysis and writing of the manuscript. The first author (CA) wrote the first draft and the other authors (SM, GZR, JH and EKW) contributed significantly to the revision of the draft.CA and EKW did the data analysis. $\mathrm{CO}$ was the senior researcher on the team for 
providing overall guidance right from protocol development to the final manuscript.All authors read and approved the final manuscript.

\section{Authors' information}

CA is a Trainee on the on the Mbarara Alzheimer's Disease Research Initiative (MADRI) - (Grant number D43TW010128: Celestino Obua)

$\mathrm{CO}$ is a senior researcher, a Pharmacologist, and Vice Chancellor of MbararaUniversity of Science and Technology. He is the Principal Investigator Grant number D43TW010128: Celestino Obua)

GZR is a Senior Lecturer in the Department of Psychiatry,Mbarara University of Science and Technology. $\mathrm{He}$ is also a key person on the Mbarara Alzheimer's Disease Research Initiative (MADRI) - (Grant number D43TW010128: Celestino Obua)

$\mathrm{JH}$ is the Director of Research at the Massachusetts General Hospital Center for Global Health and Associate Professor at Harvard Medical School. She is also a key person on the Mbarara Alzheimer's Disease Research Initiative (MADRI) - (Grant number D43TW010128: Celestino Obua)

EKW is a Research Administrator/Manager in the Office of Research Administration at Mbarara University of Science and Technology. She is also key person on the Mbarara Alzheimer's Disease Research Initiative (MADRI) - (Grant number D43TW010128: Celestino Obua)

\section{Ethics approval and consent to participate}

Ethical approval was provided by the Mbarara University Research EthicsCommittee under application number 'MUREC 1/7. The study wasthen registered with the Uganda National Council for Science and Technology under the number 'HS316ES'. All participants gave voluntarywritteninformed consent to participate in this study.

\section{Consent for publication}

Not applicable.

\section{Competing interests}

The authors declare that they have no competing interests.

\section{References}


1. Horton R. GBD 2010: understanding disease, injury, and risk. The Lancet. 2012;380(9859):2053-4.

2. Alzheimer's Disease International. World Alzheimer Report 2009. 64 Great Suffolk Street, London SE1 OBL, UK: Alzheimer's Disease International: The International Federation of Alzheimer's Disease and Related Disorders Societies, Inc. ; 2009.

3. Prince MJ, Acosta D, Castro-Costa E, Jackson J, Shaji K. Packages of care for dementia in low-and middle-income countries. PLoS Medicine. 2009;6(11):e1000176.

4. Prince M, Wimo A, Guerchet M, Ali G, Wu Y, Prina M. World Alzheimer Report 2015. The Global Impact of Dementia. Alzheimer's Disease International. Alzheimer's Disease International (ADI), London. 2015.

5. Republic of Uganda. National Population and Housing Census 2014: Area Specific Profiles. UgandaBureauofStatistics; 2017.

6. Nakasujja N, Musisi S, Walugembe J, Wallace D. Psychiatric disorders among the elderly on nonpsychiatric wards in an African setting. Int Psychogeriatr. 2007;19(4):691-704.

7. George-Carey R, Adeloye D, Chan KY, Paul A, Kolcic I, Campbell H, et al. An estimate of the prevalence of dementia in Africa: A systematic analysis. J Glob Health. 2012;2(2):020401.

8. Barrett JJ, Haley WE, Harrell LE, Powers RE. Knowledge about Alzheimer disease among primary care physicians, psychologists, nurses, and social workers. Alzheimer disease and associated disorders. 1997.

9. Pucci E, Angeleri F, Borsetti G, Brizioli E, Cartechini E, Giuliani G, et al. General practitioners facing dementia: are they fully prepared? Neurological Sciences. 2004;24(6):384-9.

10. Kalula S, Petros G. Responses to dementia in less developed countries with a focus on South Africa. IFA Global Ageing 2011; 7 (1): 31. 2011;39.

11. World Health Organization. Dementia toolkit for community workers in low-and middle-income countries: guide for community-based management and care of people with dementia. Manila: WHO Regional Office for the Western Pacific; 2018.

12. Schindel Martin L, Gillies L, Coker E, Pizzacalla A, Montemuro M, Suva G, et al. An education intervention to enhance staff self-efficacy to provide dementia care in an acute care hospital in Canada: a nonrandomized controlled study. American Journal of Alzheimer's Disease \& Other Dementias®. 2016;31(8):664-77.

13. Nsereko JR, Kizza D, Kigozi F, Ssebunnya J, Ndyanabangi S, Flisher AJ, et al. Stakeholder's perceptions of help-seeking behaviour among people with mental health problems in Uganda. International journal of mental health systems. 2011;5(1):5.

14. Ministry of Health. Uganda Clinical Guidelines- National Guidelines for Management of Common Conditions. 2016.

15. Wakida EK, Obua C, Rukundo GZ, Maling S, Talib ZM, Okello ES. Barriers and facilitators to the integration of mental health services into primary healthcare: a qualitative study among Ugandan primary care providers using the COM-B framework. BMC health services research. 2018;18(1):890. 
16. Declaration K, editor Minimum actions required for the care of people with dementia. 20th International Conference of Alzheimer's Disease International, Kyoto, Japan Kyoto, Alzheimer's Disease International; 2004.

17. Ministry of Health. Uganda Hospital and Health Centre IV Census Survey 2014. Kampala; 2014.

18. Braun V, Clarke V. Using thematic analysis in psychology. Qualitative research in psychology. 2006;3(2):77-101.

19. Muhr T. Atlas. ti: qualitative data analysis, version 7. Berlin: Scientific Software Development GmbH. 2013.

20. Montgomery et al KPPA. General practitioners' knowledge, practices, and obstacles in the diagnosis and management of dementia. Aging \& Mental Health. 2014.

21. Pathak KP, Montgomery A. General practitioners' knowledge, practices, and obstacles in the diagnosis and management of dementia. Aging \& mental health. 2015;19(10):912-20.

22. Wang M, Shao S, Li J, Liu Y, Xu X, Du J. The needs of informal caregivers and barriers of primary care workers toward dementia management in primary care: a qualitative study in Beijing. BMC family practice. 2018;19(1):201.

23. Prince $M$, Acosta D, Albanese E, Arizaga R, Ferri CP, Guerra M, et al. Ageing and dementia in low and middle income countries-Using research to engage with public and policy makers. International review of psychiatry. 2008;20(4):332-43.

24. Van Ginneken N, Tharyan P, Lewin S, Rao GN, Meera S, Pian J, et al. Non-specialist health worker interventions for the care of mental, neurological and substance-abuse disorders in low-and middleincome countries. Cochrane Database of Systematic Reviews. 2013(11).

\section{Tables}

Table 1: Study participants characteristics

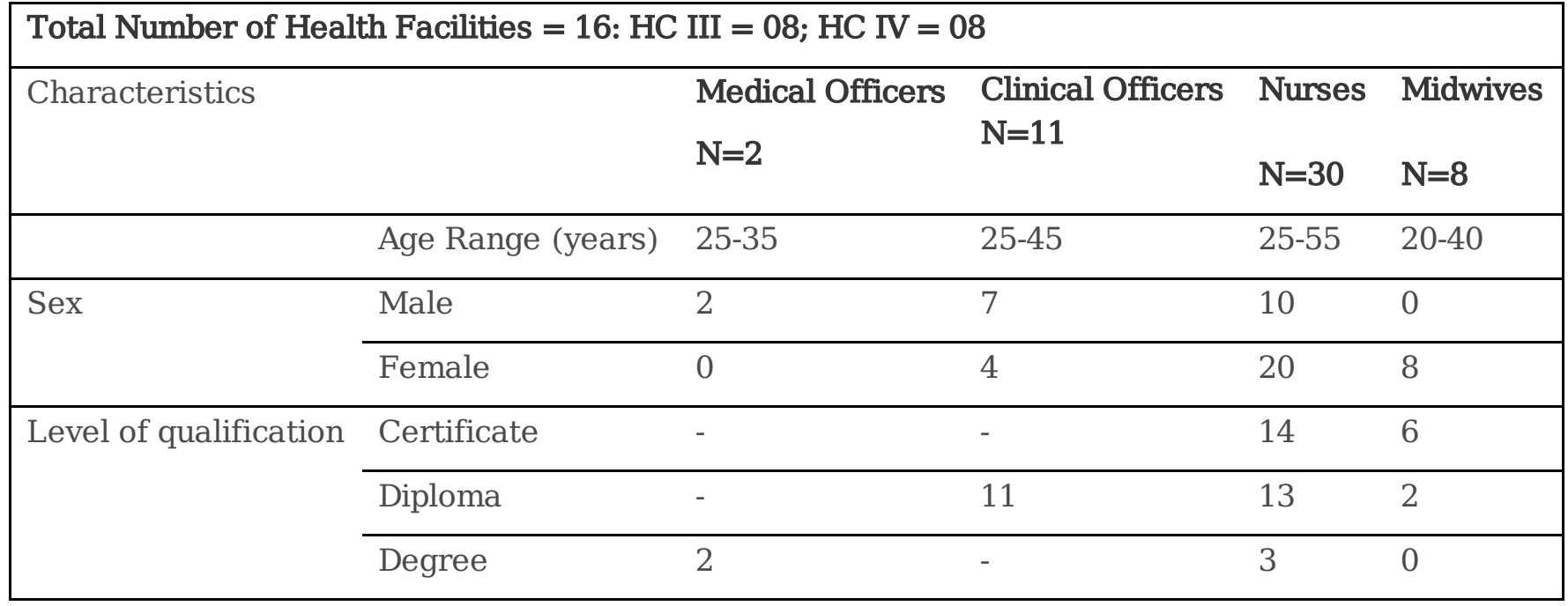

\section{Figures}




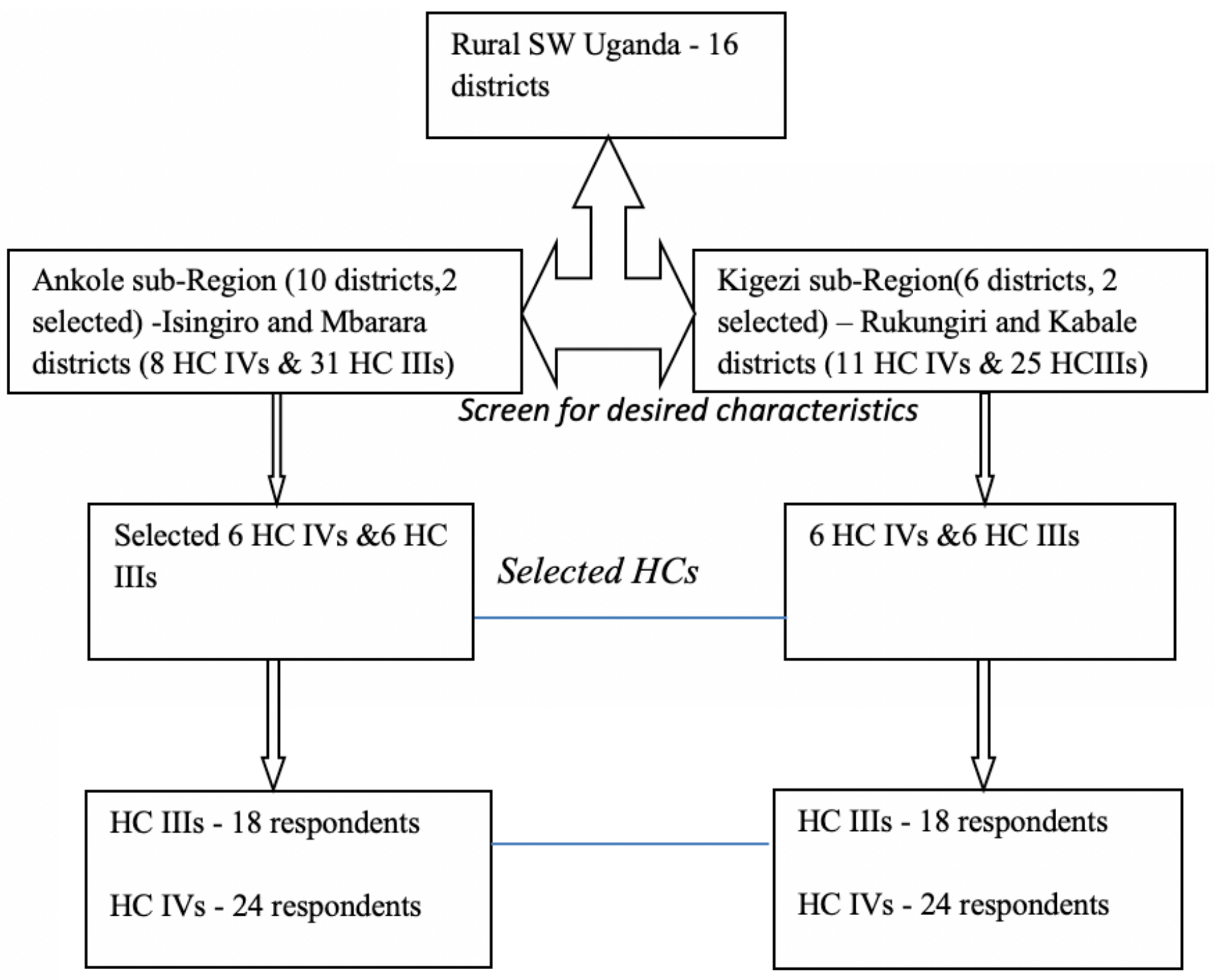

Figure 1

Sampling frame for Health Centers and Primary Healthcare Provider Participants 